ORNL/TM-13168

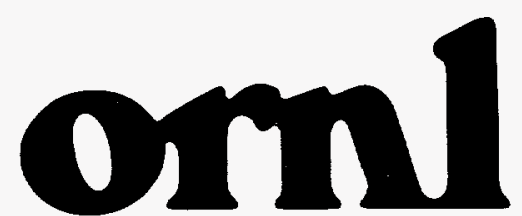

OAK RIDGE NATIONAL

LABORATORY

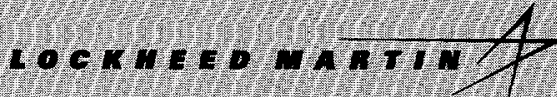

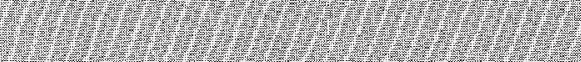

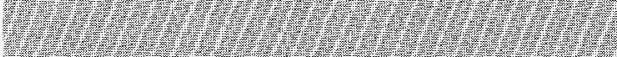

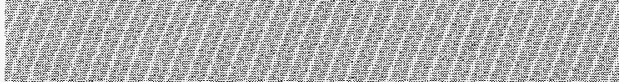
VIr

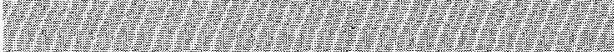

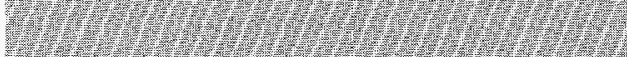
1.7. 1. Why

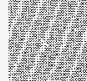

\section{MANAGED BY}

LOCKHEED MARTIN ENERGY SYSTEMS, NIC.

FOR THE UNITED STATES

DEPARTMENT OF ENERGY

\section{Min-Max Redundancy Resolution for a Mobile Manipulator}

David B. Reister 
This report has been reproduced directly from the best available copy.

Available to DOE and DOE contractors from the Office of Scientific and Technical information, P.O. Box 62, Oak Ridge, TN 37831; prices available from (423) 576-8401, FTS 626-8401.

Available to the public from the National Technical Information Service, U.S. Department of Commerce, 5285 Port Royal Rd., Springfield, VA 22161.

This report was prepared as an account of work sponsored by an agency of the United States Government. Neither the United States Government nor any agency thereof, nor any of their employees, makes any warranty, express or implied, or assumes any legal liability or responsibility for the accuracy, completeness, or usefulness of any information, apparatus, product, or process disclosed, or represents that its use would not infringe privately owned rights. Reference herein to any specific commercial product, process, or service by trade name, trademark, manufacturer, or otherwise, does not necessarily constitute or imply its endorsement, recommendation, or favoring by the United States Government or any agency thereot. The views and opinions of authors expressed herein do not necessarily state or reflect those of the United States Government or any agency thereof. 


\title{
MIN-MAX REDUNDANCY RESOLUTION for a MOBILE MANIPULATOR
}

\author{
DAVID B. REISTER
}

Research sponsored by the Engineering Research Program, Office of Basic Energy Sciences and U. S. Department of Energy

DATE PUBLISHED : FEBRUARY 1996

Prepared by

Oak Ridge National Laboratory

Oak Ridge, Tennessee 37831 managed by

Lockheed Martin Energy Research Corp.

for the

U. S. Department of Energy

under Contract No. DE-AC05-96OR22464 


\section{TABLE OF CONTENTS}

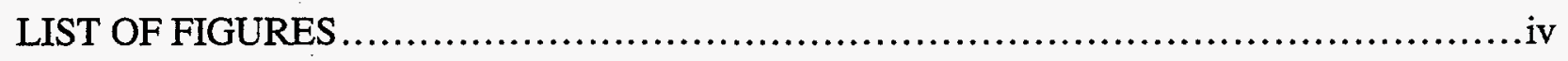

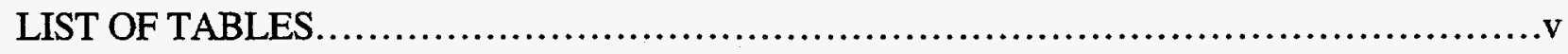

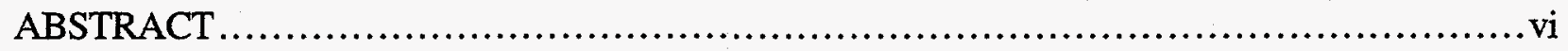

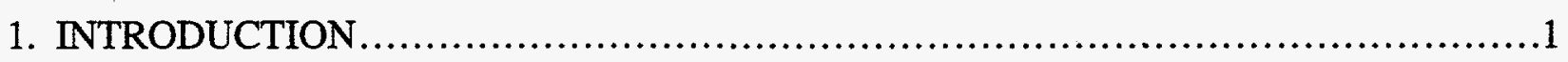

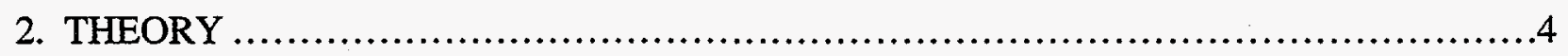

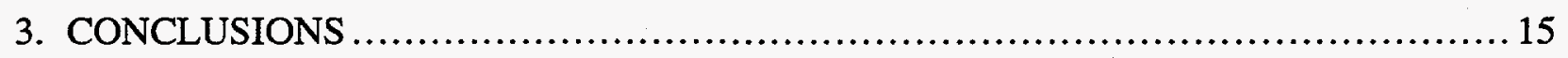

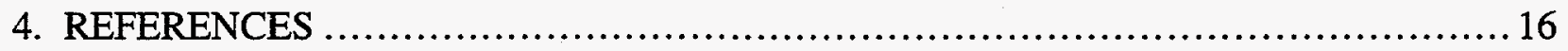




\section{LIST OF FIGURES}

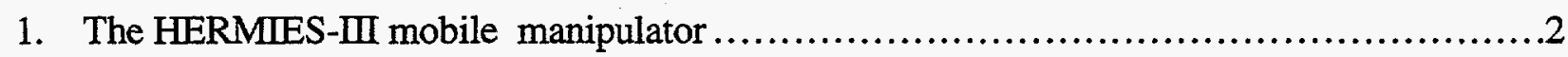

2. An optimal configuration for an objective function with distance, torque, and reach components............................................................... 11 


\section{LIST OF TABLES}

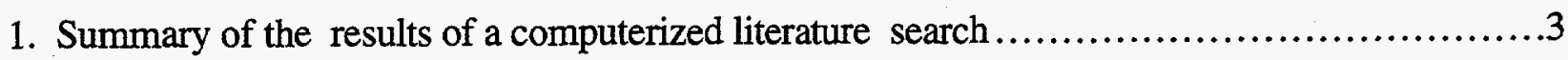

2. The five smallest distances for the third case ............................................ 12 


\section{ABSTRACT}

We have considered the problem of determining the values of the joint variables of a mobile manipulator with many redundant degrees of freedom that will minimize an objective function when the position and orientation of the end of the manipulator are given. The objective function is the weighted sum of three components: distance, torque, and reach. Each of the three components is a max or min. We have converted the min-max optimization problem into a nonlinear programming problem and used the Kuhn-Tucker conditions to derive necessary conditions for the optimum solution.

The necessary conditions require that one or more of each of the three sets (distance, torque, and reach) of nonnegative Lagrange multipliers must be positive. If one of the Lagrange multipliers is positive, the corresponding slack variable must be zero. When two or more of the Lagrange multipliers from a single set are positive, the slack variables place constraints on the joint variables. Specification of the Cartesian position and orientation of the end of the arm also places constraints on the joint variables. If the mobile manipulator has $\mathrm{N}$ degrees of freedom and the total number of constraints is $M$, the constraints define a manifold of dimension $N-M$. When $N=M$, the dimension of the manifold is zero (it consists of isolated points). When $\mathrm{N}>\mathrm{M}$, a search of the manifold may yield a submanifold that maximizes the Lagrangian function. We discuss examples where the number of slack variable constraints $(M)$ is two or more. 


\section{INTRODUCTION.}

There is great interest in mobile robots. They can carry sensors and explore the environment. But they have limited ability to change the environment. Adding a manipulator to a vehicle greatly extends the capabilities of a mobile robot. Mobile manipulators can perform many tasks: material handling, construction, and maintenance. Autonomous mobile manipulators are especially useful in environments that are hazardous to humans: battlefields, police work, handling hazardous chemicals, fire fighting, and working in high radiation fields.

We became interested in motion planning for a mobile manipulator when HERMIES-III became operational in 1989 (see Fig. 1). HERMIES-III is a large ( $820 \mathrm{~kg}$ ) mobile manipulator designed for human scale experiments. The vehicle $(1.6 \mathrm{~m} \mathrm{X} 1.3 \mathrm{~m} \mathrm{X} 1.9 \mathrm{~m})$ has two steering wheels and four corner caster wheels. The manipulator is the CESARm, a 7-DOF (degrees of freedom) compliant arm with all revolute joints, a spherical wrist, and a low friction back driveable drive train. The CESARm can reach $1.4 \mathrm{~m}$, has a load capacity of approximately $14 \mathrm{~kg}$, and has an unloaded tip speed of $3.0 \mathrm{~m} / \mathrm{s}$.

Mobile manipulators can be highly redundant. HERMIES-III combines a 3 DOF vehicle with a 7-DOF arm for a total of 10 DOF. The redundancy allows the system to both move in Cartesian space and accomplish other objectives: avoid obstacles, avoid joint limits, avoid singularities, and minimize joint torques. In three recent papers [Pin (1990), Pin (1992), and Pin (1994)], we have reported on some numerical calculations to resolve the redundancy for mobile manipulators by minimizing the maximum value of an objective function. While most methods for redundancy resolution are based on the mean square norm, we prefer the min-max norm. If each joint of the manipulator has a maximum permissible torque, we want to be sure that the torque in every joint is below the limit. We cannot be satisfied if the average torque is below the average limit. If we want to avoid obstacles, we focus on the minimum distance to an obstacle and not the average distance to an obstacle. In this paper, we define a nonlinear programming problem for the min-max problem in Pin (1994) and apply the Kuhn-Tucker conditions to derive a set of necessary conditions for the variables.

There is a large literature on algorithms for the solution of min-max problems. An example is a recent paper by Polak (1991). However, our min-max problem has an extra constraint on the joint variables [the Cartesian position and orientation of the end of the manipulator are given]. Consequently, the necessary conditions for our min-max problem are more general than for the standard problem. Polak calls a point that satisfies the necessary conditions for the standard problem a Danskin point. Our conditions will reduce to the conditions for the Danskin point when the extra constraint is removed. 


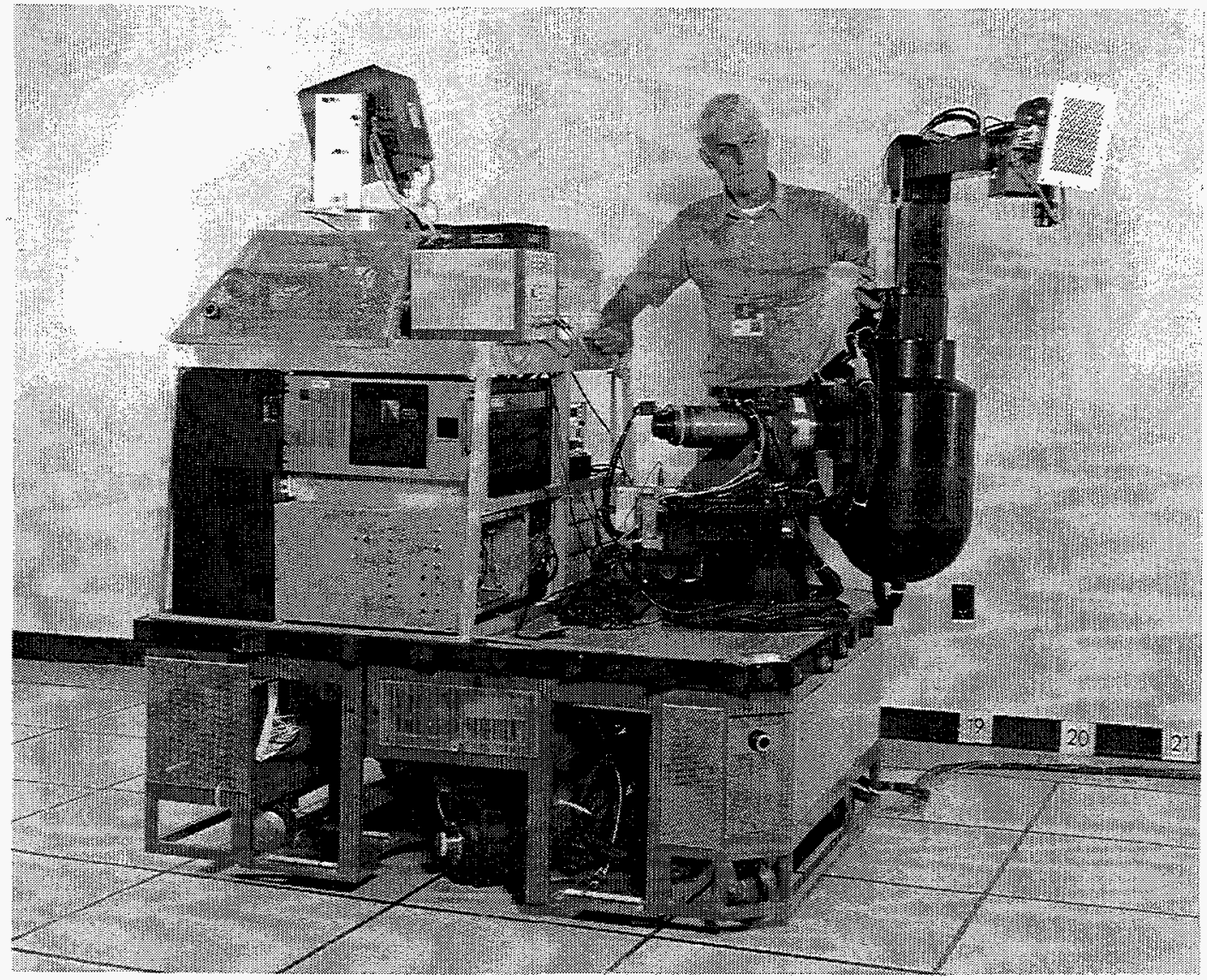

Fig. 1. The HERMIES-III mobile manipulator. 
The results of a computerized literature search are summarized in Table 1. The one paper in set S9 is our paper [Pin (1994)]. Of the 26 papers in set S8, the paper by Klein (1983) is the only one that is using the phrase min-max in the same way that we are. Klein avoids joint limits using a least squares norm and remarks that the ideal norm is the min-max norm. He claims that the minmax norm is not computationally tractable.

Table 1. Summary of the results of a computerized literature search.

\begin{tabular}{|c|c|l|}
\hline Set & Number of Papers & \multicolumn{1}{|c|}{ Keywords } \\
\hline S1 & 76,404 & Mobile (Mobile or Platform? or Vehic?) \\
\hline S2 & 42,827 & Manipulators (Manipulat? or Arm or Arms) \\
\hline S3 & 16,512 & Redundancy (Redundan?) \\
\hline S4 & 3,491 & Min-Max (Min-Max or minimax) \\
\hline S5 & 1558 & Mobile Manipulators \\
\hline S6 & 938 & Redundant Manipulators \\
\hline S7 & 77 & Redundant Mobile Manipulators \\
\hline S8 & 26 & Min-Max Manipulators \\
\hline S9 & 1 & Min-Max Mobile Manipulators \\
\hline
\end{tabular}

In the next section, we will develop a general theory for min-max redundancy resolution for mobile manipulators. Our goal is to find a trajectory in joint space that will keep the end of the manipulator on a given path and minimize a scalar objective function. The objective function is the weighted sum of three components: distance, torque, and reach. Each of the three components is a max or min: distance is the minimum distance to an object; torque is the maximum normalized joint torque; and reach is the minimum difference between the reach variable and its upper and lower limits. For torque, we have a min-max problem. For distance and reach, we have a min-min problem. We will convert the min-max optimization problem into a nonlinear programming problem and use the Kuhn-Tucker conditions to derive necessary conditions for the optimum solution. The final section will present our conclusions. 


\section{THEORY.}

When a six DOF manipulator is mounted on a three DOF vehicle, the combined system (a mobile manipulator) is highly redundant. The redundancy allows the mobile manipulator to perform a task (specified by a trajectory in Cartesian space) and simultaneously accomplish other objectives (examples include: avoid obstacles, avoid joint limits, and minimize joint torques). We will assume that the mobile manipulator is described by an $N$ vector of joint variables $(\theta)$. All points on the mobile manipulator move in Cartesian space. We are usually interested in the position and orientation of the end of the manipulator. The position and orientation of the end of the manipulator are described by a vector $(\mathbf{x})$ with six components in 3D space. There is a unique mapping from the joint space to the Cartesian space:

$$
x=\mathbf{f}(\theta)
$$

Thus, we are not considering a nonholonomic vehicle.

Our goal is to find a trajectory in joint space $[\theta(t)]$ that will keep the end of the manipulator on a given path $\left[\mathbf{x}^{\left.\left.\mathbf{G}_{(}\right)\right]}\right.$and minimize a scalar objective function $(\mathrm{L})$. The objective function has three components $\left(\mathrm{L}_{\mathrm{i}}\right)$ : distance, torque, and reach. The objective function is a weighted sum of the components:

$$
L=\sum_{i=1}^{3} w_{i} L_{i}
$$

where the $w_{i}$ are the weights. This section will use the Kuhn-Tucker conditions to derive necessary conditions for minimizing the objective function.

For distance, our objective is to avoid obstacles and not to maximize the distance from an obstacle. Thus, we consider a sphere of influence defined by its radius ( $d_{1}$ an input parameter). The minimum value of the distance component of the objective function $\left[L_{1}(d)\right]$ occurs at $d=d_{1}$, where $d$ is the distance to the closest object $[d=d(\theta)]$. When $d>d_{1}$, the objective function increases slowly and when $d<d_{1}, L_{1}$ increases strongly. When $d$ is negative, $L_{1}$ has a very large value. To be more quantative, when $d=d_{1}, L_{1}=0.01$, and when $d=d_{2}$ [where $d_{2}$ is a small positive number: $\left.\left(d_{2}=d_{1} / 1000.99\right)\right], L_{1}=1000$.

$$
\begin{array}{ll}
\mathrm{L}_{1}=0.01 \mathrm{~d} / \mathrm{d}_{1} & \text { when } \mathrm{d}>\mathrm{d}_{1} \\
\mathrm{~L}_{1}=\left(\mathrm{d}_{1} / \mathrm{d}\right)-0.99 & \text { when } \mathrm{d}_{2}<\mathrm{d}<\mathrm{d}_{1} \\
\mathrm{~L}_{1}=1000\left(1+\mathrm{d}_{2}-\mathrm{d}\right) & \text { when } \mathrm{d}<\mathrm{d}_{2}
\end{array}
$$


The static joint torque $(\tau)$ is determined by the static force and torque on the tip of the arm (F) and by the Jacobian $(\mathbf{J})$ :

$$
\tau=\mathbf{J}^{\mathbf{T}} \mathbf{F}
$$

The components of the Jacobian can be calculated from the partial derivatives of the equations relating the Cartesian position of the tip of the arm to the $\mathrm{N}$ joint variables. Given the six components of the force, the $\mathrm{N}$ components of the joint torque can be calculated $\left[\tau_{\mathrm{i}}=\tau_{\mathrm{i}}(\theta)\right]$. We would like a single number to characterize the torque state for the arm. While most research on redundancy resolution is based on the Euclidian norm, we prefer the min-max norm:

$$
Q=\max _{i}\left|\tau_{i}\right| / \tau_{i}^{m}
$$

where the $\tau_{\dot{i}}^{m}$ are limits for each joint.

Given a value for the torque norm, we need to define the torque component of the objective function $\left[\mathrm{L}_{2}(\mathrm{Q})\right]$. Our objective is to choose values for the joint variables that will reduce the torque norm but the objective function should not become very large for large values of the torque. For a given static force, we can calculate the maximum possible value for the torque norm $\left(\mathrm{Q}_{\mathrm{m}}\right)$. Our objective function is the torque norm divided by the maximum value:

$$
\mathrm{L}_{2}=\mathrm{Q} / \mathrm{Q}_{\mathrm{m}}
$$

Thus, the torque component of the objective function will be positive but less than 1.0.

The reach component of the objective function $\left[\mathrm{L}_{3}(\mathrm{D})\right]$ should be high when the arm is near singular configurations or near the boundary of its reach envelope. We define reach $(R)$ to be the distance from the base of the arm to its tip. The reach has a maximum $\left(R_{H}\right)$ and a minimum $\left(R_{L}\right)$ value; for the CESARm: $R_{H}=1.250$ meters and $R_{L}=0.277$ meters.

The reach objective function is near 98 when the reach approaches its upper or lower limit and is 0 when the reach is half way between the two limits. Let $D$ be the minimum distance between the reach and its limits: $D_{L}=R-R_{L}, D_{H}=R_{H}-R$, and $D=\min \left(D_{L}, D_{H}\right)$. Define the parameters $R_{1}$ and $R_{2}$ by: $R_{1}=R_{H}-R_{L}$ and $R_{2}=1 / 0.51$. We define the reach objective function by:

$$
L_{3}=R_{1} /\left(D+0.01 R_{1}\right)-R_{2}
$$


When $\mathrm{D}=0, \mathrm{~L}_{3}=100-\mathrm{R}_{2}$, and when $\mathrm{D}=\mathrm{R}_{1} / 2, \mathrm{~L}_{3}=0$.

The objective function has three components that depend on distance (d), torque (Q), and reach (D). We have completed our definition of the three components. Next we will relate distance, torque, and reach to the joint variables. We begin with distance.

We subdivide the mobile manipulator and the obstacles into objects. We assume that a distance function can calculate the minimum distance between each member (j) of the mobile manipulator set and each member $(\mathrm{k})$ of the obstacle set. For convenience, we make a one dimensional array (i) out of the two dimensional array $(j, k)$. Thus, $d_{i}$ is the minimum distance between some member [j(i)] of the mobile manipulator set and some member $[\mathrm{k}(\mathrm{i})]$ of the obstacle set. The minimum distance between two objects depends on the joint variables: $d_{i}=d_{i}(\theta)$. Let $d$ be the minimum distance between one component of the mobile manipulator and one component of the obstacles:

$$
\mathrm{d}=\min _{i} \mathrm{~d}_{\mathrm{i}}(\theta)
$$

Define a non-negative slack variable $\left(\rho_{i}\right)$ that is the difference between each distance $\left(d_{i}\right)$ and the minimum distance:

$$
\mathrm{d}=\mathrm{d}_{\mathrm{i}}-\rho_{\mathrm{i}} \quad \text { where } \rho_{\mathrm{i}} \geq 0
$$

Define the normalized torque $\left[\mathrm{G}_{\mathrm{i}}(\theta)\right]$ to be the magnitude of the torque at each joint divided by the torque limit for the joint:

$$
\mathrm{G}_{\mathrm{i}}(\theta)=\left|\tau_{\mathrm{i}}\right| / \tau_{\mathrm{i}}^{\mathrm{m}}
$$

Using our new notation, Eq. (7) may be written:

$$
\mathrm{Q}=\max _{i} G_{i}(\theta)
$$

Define a non-negative slack variable $\left(\sigma_{\mathfrak{i}}\right)$ that is the difference between each normalized torque $\left(\mathrm{G}_{\mathrm{i}}\right)$ and the maxmum normalized torque:

$$
\mathrm{Q}=\mathrm{G}_{\mathrm{i}}(\theta)+\sigma_{\mathrm{i}} \quad \text { where } \sigma_{\mathrm{i}} \geq 0
$$


The goal is to minimize the objective function, while placing the end of the arm at a Cartesian position $\left(\mathbf{x}^{*}\right)$ :

$$
\mathbf{f}(\theta)=\mathbf{x}^{*}
$$

Since $D$ is the minimum distance between the reach and its limits $\left[D=\min \left(D_{L}, D_{H}\right)\right]$, we can define two non-negative slack variables $\left(\psi_{i}\right)$ that are the difference between $D_{L}$ and $D_{H}$ and $D$ :

$$
\begin{array}{ll}
\mathrm{D}=\mathrm{D}_{\mathrm{H}}(\theta)-\psi_{1} & \text { where } \psi_{1} \geq 0 \\
\mathrm{D}=\mathrm{D}_{\mathrm{L}}(\theta)-\psi_{2} & \text { where } \psi_{2} \geq 0
\end{array}
$$

Using the definitions of $D_{L}$ and $D_{H}$, Eqs. (15) and (16) may be written:

$$
\begin{aligned}
& \mathrm{D}=\mathrm{R}_{\mathrm{H}}-\mathrm{R}(\theta)-\psi_{1} \\
& \mathrm{D}=\mathrm{R}(\theta)-\mathrm{R}_{\mathrm{L}}-\psi_{2}
\end{aligned}
$$

Our goal is to minimize the objective function $(\mathrm{L})$ subject to constraints on distance (d), torque (Q), and reach (D). We maximize a Lagrangian function $(\mathrm{H})$ that includes the objective function and the constraints:

$$
\begin{aligned}
& H=-w_{1} L_{1}(d)-w_{2} L_{2}(Q)-w_{3} L_{3}(D)-\sum_{i} \lambda_{i}\left(d+\rho_{i}-d_{i}(\theta)\right)+ \\
& \sum_{j} \delta_{j}\left(Q-\sigma_{j}-G_{j}(\theta)\right)-\gamma_{1}\left(D-R_{H}+R(\theta)+\psi_{1}\right)+ \\
&-\gamma_{2}\left(D-R(\theta)+R_{L}+\psi_{2}\right)+\sum_{n} \mu_{n}\left(f_{n}(\theta)-x_{n}^{*}\right)
\end{aligned}
$$

where $\lambda_{i}, \delta_{i}, \gamma_{i}$, and $\mu_{i}$ are Lagrange multipliers. The Lagrangian function depends on the joint variables, the distance, torque, and reach variables, the slack variables, and the Lagrange multipliers:

$$
\mathrm{H}=\mathrm{H}(\mathrm{d}, \mathrm{Q}, \mathrm{D}, \lambda, \rho, \theta, \delta, \sigma, \gamma, \psi, \mu)
$$


To derive a set of necessary conditions for the variables, we apply the Kuhn-Tucker conditions to the Lagrangian function. For the unrestricted variables (d, $Q, D, \theta, \lambda, \delta, \gamma$, and $\mu$ ), the Kuhn-Tucker conditions require that the partial derivatives of $H$ with respect to each variable is zero. For the slack variables $(\rho, \sigma$, and $\psi$ ), the Kuhn-Tucker conditions require that the partial derivatives of $\mathrm{H}$ with respect to each variable is non-positive and the sum of the Lagrange multipliers and the slack variables equals zero. For our problem, the necessary conditions are:

$$
\begin{aligned}
& \sum_{i} \lambda_{i}=-w_{1} \frac{\partial L_{1}}{\partial d} \\
& \sum_{i} \delta_{i}=w_{2} \frac{\partial L_{2}}{\partial Q} \\
& \sum_{i} \gamma_{i}=-w_{3} \frac{\partial L_{3}}{\partial D}
\end{aligned}
$$

$\sum_{i} \lambda_{i} \frac{\partial \mathrm{d}_{\mathrm{i}}}{\partial \theta_{\mathrm{m}}}-\sum_{\mathrm{j}} \delta_{j} \frac{\partial \mathrm{G}_{\mathrm{i}}}{\partial \theta_{\mathrm{m}}}-\left(\gamma_{1}-\gamma_{2}\right) \frac{\partial \mathrm{R}}{\partial \theta_{\mathrm{m}}}+\sum_{\mathrm{n}} \mu_{\mathrm{n}} \frac{\partial \mathrm{f}_{\mathrm{n}}}{\partial \theta_{\mathrm{m}}}=0$

$\lambda_{\mathrm{i}} \geq 0$

$$
\sum_{i} \lambda_{i} \rho_{i}=0
$$

$\delta_{\mathrm{i}} \geq 0$

$$
\sum_{i} \delta_{i} \sigma_{i}=0
$$

$\gamma_{i} \geq 0$

$$
\sum_{\mathrm{i}} \gamma_{\mathrm{i}} \psi_{\mathrm{i}}=0
$$


The necessary conditions place restrictions on the signs of the derivatives of the components of the objective function. Equations (22) to (24) and the conditions that the Lagrange multipliers are non-negative require that the components of the objective function satisfy:

$$
\begin{aligned}
& \frac{\partial \mathrm{L}_{1}}{\partial \mathrm{d}} \leq 0 \\
& \frac{\partial \mathrm{L}_{2}}{\partial \mathrm{Q}} \geq 0 \\
& \frac{\partial \mathrm{L}_{3}}{\partial \mathrm{D}} \leq 0
\end{aligned}
$$

Since $L_{1}$ increases when $d>d_{1}$ and decreases when $d<d_{1}$, inequality (32) is satisfied for $\mathrm{d}_{2}<\mathrm{d}<\mathrm{d}_{1}$. Inequality (33) is satisfied because $\mathrm{L}_{2}$ increases with $\mathrm{Q}$ [see Eq. (8)]. Inequality (34) is satisfied because $\mathrm{L}_{3}$ decreases with $\mathrm{D}$ [see Eq. (9)].

Since the right sides of Eqs. (22) to (24) are positive, one or more of each of the Lagrange multipliers $\left(\lambda_{i}, \delta_{i}\right.$, and $\left.\gamma_{i}\right)$ must be positive. Equations (27), (29), and (31) require that sums of the products of Lagrange multipliers and slack variables must be zero. Since both the Lagrange multipliers and slack variables are non-negative, every term in the sums must be zero. If one of the Lagrange multipliers is positive (for example, $\lambda_{\mathrm{n}}$ ), the corresponding slack variable must be zero (for example, $\rho_{n}$ ). If two of the Lagrange multipliers for distance are positive, then two of the corresponding distance slack variables must be zero: $d=d_{1}(\theta)$ and $d=d_{2}(\theta)$.

When the two reach slack variables $\left(\psi_{\mathrm{i}}\right)$ are zero, Eqs. (18) and (19) imply that $\mathrm{R}(\theta)=\left(\mathrm{R}_{\mathrm{H}}+\mathrm{R}_{\mathrm{L}}\right) / 2$. Furthermore, $\mathrm{L}_{3}=0$.

When two or more of the distance slack variables must be zero, the joint variables are constrained:

$$
\mathbf{g}(\theta)=\mathbf{0}
$$

where $\mathbf{g}$ is a vector with $M$ components. Let $\mathbf{J}_{\mathbf{g}}$ be the Jacobian matrix of $\mathbf{g}$ :

$$
\mathbf{J}_{\mathbf{g}}=\frac{\partial \mathbf{g}(\theta)}{\partial \theta}
$$


If the rank of $J_{g}$ is $M$, Eq. (35) defines a manifold of dimension $\mathbf{N}-\mathbf{M}$. Specification of the Cartesian position and orientation of the end of the arm [Eq. (15)] places constraints on the joint variables. When two or more of the slack variables are zero, Eqs. (11), (14), and (18) and (19) place constraints on the joint variables. When $\mathrm{N}=\mathrm{M}$, the dimension of the manifold is zero (it consists of isolated points). When $N>M$, a search of the manifold may yield a submanifold that maximizes the Lagrangian function $(\mathrm{H})$.

In Pin (1994), we used numerical search techniques to maximize the Lagrangian function $(\mathrm{H})$. Results were obtained for three cases. Since the goal $\left(\mathbf{x}^{*}\right)$ is a Cartesian position but not an orientation, the goal provides three constraints. The mobile manipulator has $3 \mathrm{DOF}$ for the vehicle and 4 DOF for the manipulator (thus, $N=7$ ). For the first case [Fig. 5 in Pin (1994)], the weights for reach and torque were zero and the Cartesian position for the wrist of the manipulator was 0.15 meters above the top of a box. For the best solution that we found numerically, only one of the slack variables was zero for distance, torque, and reach. Thus, one distance was minimum, one torque was maximum, and the reach was not at the mid-point $\left[\left(\mathrm{R}_{\mathrm{H}}+\mathrm{R}_{\mathrm{L}}\right) / 2\right]$.

For the second case [Fig. 6 in Pin (1994)], all three components of the objective function were active $\left(w_{i}=1\right.$ for $\left.i=1,2,3\right)$ and the Cartesian position for the wrist of the manipulator remained at 0.15 meters above the top of a box. For the best solution that we found numerically, only one of the slack variables was zero for distance and torque. Since the reach was at the midpoint, the two reach slack variables $\left(\psi_{\mathrm{i}}\right)$ were zero.

For the third case [Fig. 7 in Pin (1994) and Fig. 2 in this paper], all three components of the objective function were active $\left(w_{i}=1\right.$ for $i=1,2,3$ ) and the Cartesian position for the wrist of the manipulator was surrounded by four boxes. For the best solution that we found numerically, only one of the slack variables was zero for torque and reach. As shown in Table 2, several of the distances were almost equal. In Table 2, the first two distances are equal. By further searching, we might find joint variables that would make the first three distances equal. It is possible that we might find joint variables that would make the first five distances equal. If we had five distance slack variables that were zero, we would have four distance constraints and three Cartesian constraints and might have $\mathrm{N}=\mathrm{M}=7$. 


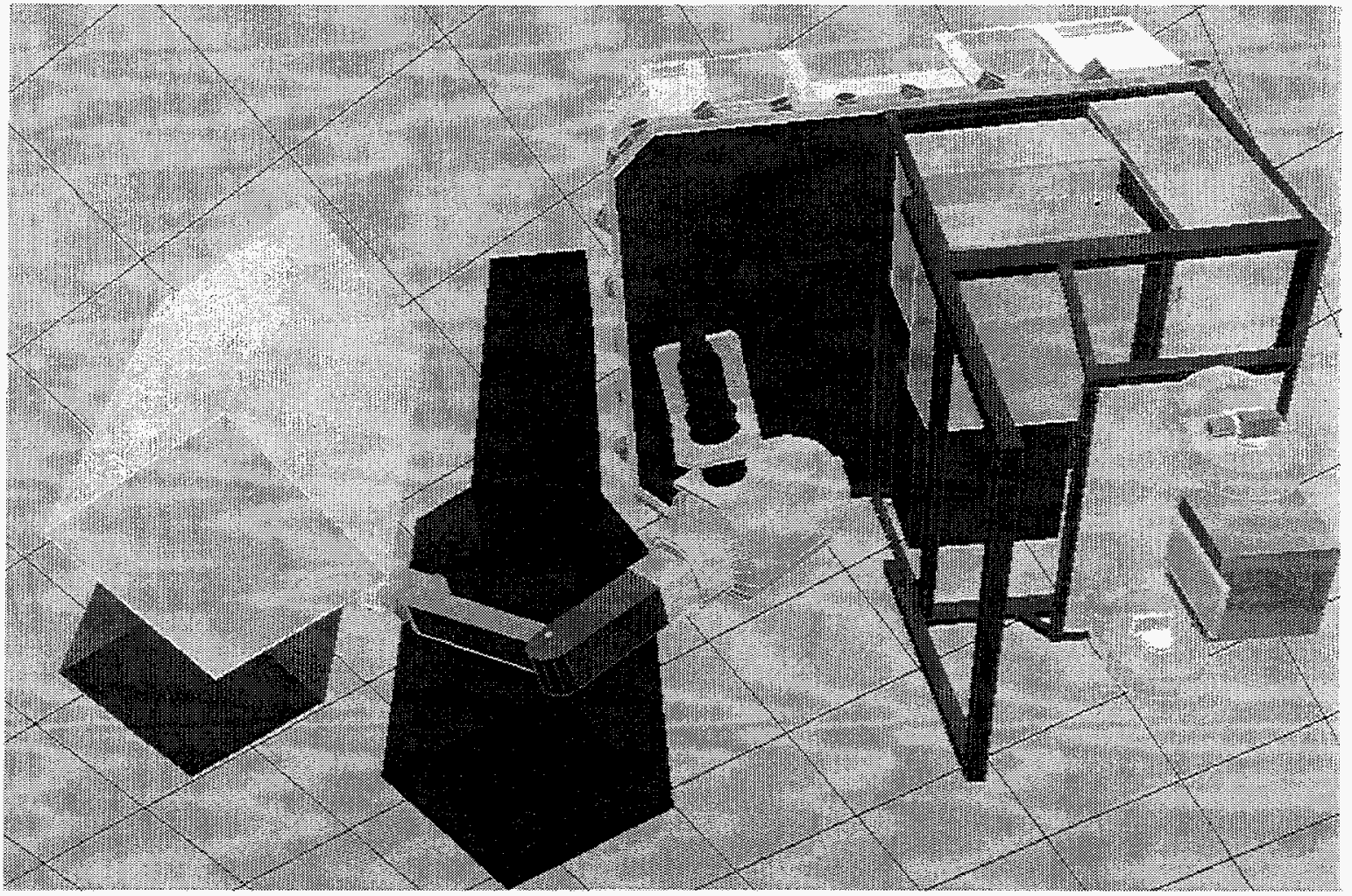

Fig. 2. An optimal configuration for an objective function with distance, torque, and reach components. 
Table 2. The five smallest distances for the third case.

\begin{tabular}{|l|l|}
\hline$i$ & $d_{i}$ \\
\hline 1 & 0.049143 \\
\hline 2 & 0.049148 \\
\hline 3 & 0.050254 \\
\hline 4 & 0.052344 \\
\hline 5 & 0.053112 \\
\hline
\end{tabular}

To simplify Eq. (25), we will group the functions for distance, torque, and reach into a single vector $[\mathbf{h}(\theta)]$ :

$$
\mathbf{h}(\theta)=\left[\begin{array}{c}
-\mathbf{d} \\
\mathbf{G} \\
\mathbf{R} \\
-\mathbf{R}
\end{array}\right]
$$

We will group the Lagrange multipliers into a single vector $[\alpha]$ :

$$
\alpha=\left[\begin{array}{l}
\lambda \\
\delta \\
\gamma
\end{array}\right]
$$

Using our two new vectors, Eq. (25) becomes:

$$
\mathbf{J}_{\mathbf{h}}^{\mathbf{T}} \alpha=\mathbf{J}^{\mathbf{T}} \mu
$$

where $\mathbf{J}_{\mathbf{h}}$ is the Jacobian matrix of $\mathbf{h}$ :

$$
\mathbf{J}_{\mathbf{h}}=\frac{\partial \mathbf{h}(\theta)}{\partial \theta}
$$

Equation (39) is our generalization of the conditions for a Danskin point. If we ignore the constraint on the Cartesian position and orientation of the end of the arm [Eq. (15)], $\mu=0$ and Eq. (39) reduces to Polak's condition [his Eq. (7)]. Only a few of the components of the nonnegative $\alpha$ vector will be positive (most of them will be zero). For each component that is positive, the corresponding slack variable will be zero. To satisfy Polak's condition, a linear combination of a 
few of the columns of $\mathbf{J}_{\mathbf{h}}^{\mathbf{T}}$ must map to an $\mathbf{N}$ component zero vector. Let $\mathbf{V}$ be a basis for the null space of $\mathbf{J}$. Since the columns of $\mathbf{J}^{\mathbf{T}}$ and $\mathbf{V}$ form a basis for the space of all vectors with $\mathbf{N}$ components, any vector with $\mathrm{N}$ components (u) can be written:

$$
\mathbf{u}=\mathbf{J}^{\mathbf{T}} \mu+\mathbf{V} \boldsymbol{\beta}
$$

The right side of Eq. (39) does not have the null space term. To satisfy our condition, a linear combination of a few of the columns of $\mathbf{J}_{\mathbf{h}}^{\mathbf{T}}$ must map to an $\mathrm{N}$ component vector that is in the subspace spanned by the columns of $\mathbf{J}^{\mathbf{T}}$ and does not have a projection in the null space of $\mathbf{J}$.

We can classify the sets of points that satisfy the necessary conditions based on the number of the $\alpha_{i}$ that are positive. For Class 1, one of the $\alpha_{i}$ will be positive. For Class 2 , two of the $\alpha_{i}$ will be positive. For Class $n, n$ of the $\alpha_{i}$ will be positive.

We have developed a general theory for min-max redundancy resolution for mobile manipulators. In Reister (1993), we have developed numerical examples for less general problems. The objective function has only one component: torque. Furthermore, the vector for position and orientation has one component $(z)$. The necessary conditions require that ane or more of the joints the magnitude of the normalized torques will be equal to the min-max value. We have classified the sets of points that satisfy the necessary conditions based on the number of the normalized torques that are equal to the min-max value. We have solved the min-max problem by identifying all of the points in all of the classes. We have explored the torque surfaces for two mobile manipulators: a planar manipulator and the CESARm. The mobile planar manipulator has three revolute joints. We began by exploring the Class 3 paths. We defined four cases and 16 subcases. For each of the 16 subcases, we have defined the three link angles in terms of a single parameter. By relating the three link angles to the parameter, we have resolved the redundancy.

We have found that 13 of the 16 subcases satisfy the necessary conditions. Of the 13 subcases, there are five configurations with a one meter workspace, three configurations with a three meter workspace, and one configuration with a five meter workspace. Given an initial value for $\mathrm{z}$ and a final value, a path planner will choose one of the 13 options. All but one of the options has a portion of the workspace where it is superior to any of the other options. Thus, the Class 3 paths reach all parts of the workspace.

We have explored Class 2 and Class 1 paths. We have surveyed Class 2 paths for a constant value of $\mathrm{z}$. By examining Class 2 paths, we have been able to identify all 10 of the Class 3 subcases that can reach the point $z=0.25$. In 8 of the 10 subcases, we have found a local minima. All of the local minima for the Class 2 paths occurred at Class 3 points (where the torques were equal at all three joints). Thus, we were unable to find any Class 2 local minima. All of the Class 1 solutions had higher values for the torque than the best Class 3 paths. 
The CESARm is a manipulator with 7 degrees of freedom that can reach points in 3D space [Dubey (1991)]. We assumed that the CESARm is mounted on a mobile vehicle that will control the $\mathrm{x}$ and $\mathrm{y}$ coordinate of the arm's tip We have three joint angles controlling the height of the arm (z). We explored the Class 3 paths. There are four ways that three torques can be equal. For the planar manipulator, the four cases were distinct. For the CESARm, the four cases correspond to changes of variables. We found four segments for the Class 3 paths. While the four segments have low values for the maximum torque, they only cover part of the workspace and do not join together.

By exploring Class 2 paths, we have found paths that cover the workspace and bridge between the disjoint Class 3 path segments. We have evaluated the necessary conditions for both the Class 3 paths and the Class 2 paths. In most cases, the paths satisfy the necessary conditions.

We have examined all of the possible Class 1 solutions. In all cases, the Class 1 solutions have much higher values for the torque than the best Class 2 and Class 3 paths. 


\section{CONCLUSIONS}

We have considered the problem of determining the values of the joint variables of a mobile manipulator with many redundant degrees of freedom that will minimize an objective function when the position and orientation of the end of the manipulator are given. The objective function is the weighted sum of three components: distance, torque, and reach. Each of the three components is a max or min. We have converted the min-max optimization problem into a nonlinear programming problem and used the Kuhn-Tucker conditions to derive necessary conditions for the optimum solution.

The necessary conditions require that one or more of each of the three sets (distance, torque, and reach) of nonnegative Lagrange multipliers must be positive. If one of the Lagrange multipliers is positive, the corresponding slack variable must be zero. When two or more of the Lagrange multipliers from a single set are positive, the slack variables place constraints on the joint variables. Specification of the Cartesian position and orientation of the end of the arm also places constraints on the joint variables. If the mobile manipulator has $\mathrm{N}$ degrees of freedom and the total number of constraints is $M$, the constraints define a manifold of dimension $N-M$. When $N=M$, the dimension of the manifold is zero (it consists of isolated points). When $N>M$, a search of the manifold may yield a submanifold that maximizes the Lagrangian function. We have discussed examples where the number of slack variable constraints $(M)$ is two or more. 


\section{REFERENCES}

1. Dubey, R. V., J. A. Euler, and S. M. Babcock, "Real-Time Implementation of an Optimization Scheme for Seven-Degree-of-Freedom Redundant Manipulators," IEEE Transactions on Robotics and Automation, 579-588 (1991).

2. Klein, C. A. and C. H. Huang, "Review of Pseudoinverse Control with Kinematically Redundant Manipulators," IEEE Transactions on Systems, Man, and Cybernetics, 245250 (1983).

2. Pin, F. G. and J. C. Culioli, "Optimal Positioning of Redundant Manipulator-Platform Systems for Maximum Task Efficiency," Robotics and Manufacturing, 3, 489-495 (1990)

3. Pin, F. G. and J. C. Culioli, "Optimal Positioning of Combined Mobile Platform Manipulator Systems for Material Handling Tasks," Journal of Intelligent and Robotic Systems, 6, 165-182 (1992).

4. Pin, F. G. J. C. Culioli and D. B. Reister, "Using Minimax Approaches to Plan Optimal Task Commutation Configurations for Combined Mobile Platform-Manipulator Systems," IEEE Transactions on Robotics and Automation, 10, 44-54 (1994).

5. Polak, E., D. Q. Mayne, and J. E. Higgins, "Superlinearly Convergent Algorithm for MinMax Problems," Journal of Optimization Theory and Applications, 69, 407-439 (1991).

6. Reister, D. B., "Using Min-Max of Torque to Resolve Redundancy for a Mobile Manipulator," ORNL/TM-12489, Oak Ridge National Laboratory (1993). 


\section{INTERNAL DISTRIBUTION}

1-5. J. Barhen

6. M. Beckerman

7. C. W. Glover

8-12. W. C. Grimmell

13. J. P. Jones

14. H. E. Knee

15-19. R. C. Mann

20. M. D. Morris

21. E. M. Oblow

22-26. C. E. Oliver

27. L. E. Parker

28. V. Protopopescu
29. N. S. V. Rao

30-34. D. B. Reister

35. S. Shekhar

36-40. R. F. Sincovec

41. E. C. Uberbacher

42. M. A. Unseren

43. CSMD Reports Office

44-45. Laboratory Records Department

46. Laboratory Records, ORNL:-RC

47. Document Reference Section

48. Central Research Library

49. ORNL Patent Office

\section{EXTERNAL DISTRIBUTION}

50. Dr. Fred Aminzadeh, 40 Paseo Estrella, Anaheim Hills, CA 92807

51. Dr. John Baillieul, Aerospace and Mechanical Engineering Department, Boston University, 110 Cummington St., Boston, MA 02215

52. Dr. John Blair, JBX Technologies, 25 Moore Road, Wayland, MA 01778

53. Professor Roger W. Brockett, Harvard University, Pierce Hall, 29 Oxford St., Cambridge, MA 02138

54. Dr. Oscar P. Manley, Division of Engineering, Mathematical, and Geosciences, Office of Basic Energy Sciences, ER-15, U.S.

Department of Energy, Germantown, Washington, DC 20545

55. Dr. K. S. Narendra, Yale University, Center for Systems Science, Department of Electrical Engineering, P.O. Box 208267, New Haven, CT 06520-8267

56. Dr. Wes Snyder, Department of Radiology, Bowman Gray School of Medicine, N.C. Baptist School of Medicine, 300 S. Hawthorne Dr., Winston-Salem, NC 27103

57. Professor Mary F. Wheeler, Department of Mathematical Sciences, Rice University, P.O. Box 1892, Houston, TX 77251

58. Office of Assistant Manager for Energy Research and Development, U.S. Department of Energy Ridge Operations Office, P.O. Box 2001, Oak Ridge, TN 37831-8600

59-60. Offic of Scientific \& Technical Information, P.O. Box 62, Oak Ridge, TN 37830 